\title{
Prognostic risk factors for patients with hepatic sinusoidal obstruction syndrome caused by pyrrolizidine alkaloids
}

Xiaofei Du ( $\nabla$ duxiaofei@ccmu.edu.cn )

Capital Medical University https://orcid.org/0000-0003-3719-2141

Zhenli Liu

Capital Medical University

Haibin Yu

Capital Medical University

Yu Wang

Beijing Tongren Hospital

Zhengsheng zou

the fifth medical center of PLA general hospital

Hongshan Wei

beijing ditan hospital

Jing Liang

Tianjin Third Central Hospital

Daokun Yang

The First Affiliated Hospital of xinxiang Medical University

Yali Liu

Capital Medical University

Jing Zhang

Capital Medical University https://orcid.org/0000-0002-3082-8330

Calvin Q. Pan

NYU grossman school of medicine

\section{Research Article}

Keywords: Sinusoidal obstruction syndrome, Pyrrolizidine alkaloids, Anticoagulant, Prognosis factors, Case fatality, Survival

Posted Date: April 20th, 2021

DOl: https://doi.org/10.21203/rs.3.rs-359053/v1 
License: (c) (i) This work is licensed under a Creative Commons Attribution 4.0 International License. Read Full License 


\section{Abstract}

Background Pyrrolizidine alkaloids induced hepatic sinusoidal obstruction syndrome (PA-HSOS) often occurs after consuming herbs and a dietary supplement containing the plant Tu-San-Qi. Limited data exists to identify patients with fatal outcomes for early interventions. We aimed to analyze the predictors for 3-month survival.

Methods We retrospectively enrolled PA-HSOS patients in 5 hospitals and extracted data from the onset of PA-HSOS to 36 months. Outcome measurements were 3-month and 36-month survival rates, baseline prognostic predictors for survival, and the effects of anticoagulant therapy.

Results Among 49 enrollees, the median age was 60 and $49 \%$ male. At the onset of PA-HSOS, patients with Child-Turcotte-Pugh (CTP) class of A, B, or C were 8.2\% (4/49), 42.8\% (21/49), and 49.0\% (24/49), respectively. None of them received a transjugular intrahepatic portosystemic shunt or a liver transplant. The 3-month and 36-month survival rates were $86 \%$ and $75 \%$, respectively. Compared to the CTP class A or $\mathrm{B}$, class $\mathrm{C}$ at baseline independently predicted lower survival rates at both 3 and 36 months. However, anticoagulation therapy treatment within the first three months independently predicted significantly higher survival rates at both time-points. The greatest benefit of anticoagulants (HR=2.54) was in patients with CTP class C compared with the survival rates with those without treatment $(93 \%$ vs. $40 \%$, $p=0.009)$. There were no bleeding complications reported in patients treated with the anticoagulant.

Conclusion We observed a 3-month survival rate of $86 \%$ in PA-HSOS patients, CTP class $\mathrm{C}$ and receiving anticoagulant therapy were the independent predictors associated with the survival.

\section{Introduction}

Hepatic sinusoidal obstruction syndrome (HSOS) is a rare type of liver injury characterized morphologically by a loss of sinusoidal wall integrity ${ }^{1}$. The most common causes are myeloablative regimens used in hematopoietic stem cell transplantation (HSCT). Other etiologies include chemotherapeutic drugs or immunosuppressive agents for solid tumors, total body or hepatic irradiation, platelet transfusion containing ABO-incompatible plasma, and consuming poisonous plants ${ }^{1}$. The typical manifestations are weight gain, jaundice, and ascites. The onset of HSOS often worsens the underlining disease's outcomes ${ }^{2,3}$. Although HSOS caused by poisonous plants has been well documented, the clinical spectrum and outcomes have not been investigated extensively ${ }^{1}$. It is estimated that more than 6000 plants contain pyrrolizidine alkaloids (PA), which caused at least 8000 cases of HSOS worldwide 4 . In China, the main plant containing PA is named "Tu-San-Qi", which is often mistaken as 'San-qi' due to their similar appearance. As 'San-qi' is a widely used medicinal preparation and herbal health product, PAinduced HSOS (PA-HSOS) often occurs in patients who have consumed "Tu-San-Qi" instead of "San-qi". Subsequently, the PA metabolites damage hepatic endothelial cells, following by hepatic congestion, liver injury, and portal hypertension ${ }^{5}$. 
Currently, data are limited on the disease presentation, diagnosis, and particularly regarding risk factors for predicting outcomes in patients with PA-HSOS. In a review by Zhuge et al, the common presentations of PA-HSOS were reported as hepatomegaly, ascites, right upper quadrant pain, jaundice, and abnormal liver transaminases ${ }^{6}$. Upper gastrointestinal bleeding and hepatic failure were found as the major contributors to mortality, which was estimated in a range from $12.2-78.6 \%^{6}$. However, only one study by Wang et al. analyzed the prognostic predictors for the survival in PA-HSOS. Although hepatic encephalopathy, serum bilirubin levels, and albumin levels were identified as independent factors for short-term mortality $7,70 \%$ of their patients were treated with an anticoagulant, followed by a transjugular intrahepatic portosystemic shunt (TIPSS) if cases were severe. Therefore, the cohort analysis was largely a study on outcomes of patients on anticoagulants with or without TIPSS therapy. In addition, there was no baseline comparison between patients treated with and without anticoagulants. In terms of treatment, all severe PA-HSOS could receive liver transplantation after the failure of medical interventions. However, it is difficult to overcome the challenge of persistent organ shortage and increasing deaths on the waiting list. For mild to moderate liver injury from PA-HSOS, several studies suggested treatment with anticoagulants or TIPSS but results remained conflicting ${ }^{6-9}$. With that in mind, we designed a retrospective cohort study and aimed to investigate the impact of clinical factors including the use of anti-coagulant on the short-term survival of patients with PA-HSOS. Our data can potentially help clinicians with identifying patients who have a higher risk of mortality and gaining a better understanding of the value of anticoagulants in managing PA-HSOS.

\section{Methods}

The present research was a multi-center retrospective study that enrolled patients from 5 tertiary care centers and hospitals located in the North and Middle regions of China during the period between $1 / 1 / 2012$ and $7 / 31 / 2018$. The study protocol was approved by the individual Ethics Review Committee in all participated research sites including the leading institution, Beijing Youan Hospital (IRB approval number [2020]-233). Patients' informed consent was exempted by IRB.

\section{Patient selection and enrollment criteria}

Although there are no international guidelines available for the diagnosis of PA-HSOS, the following diagnostic criteria of HSCT-induced HSOS have been recommended by the European Society for Blood and Marrow Transplantation (EBMT) ${ }^{10}$ : Within 21 days after HSCT, HSOS is diagnosed based on bilirubin $\geq 2 \mathrm{mg} / \mathrm{dL}$ plus any two of the three features including weight gain $>5 \%$, ascites, or painful hepatomegaly. Late-onset of HSOS >21 days after HSCT can be diagnosed with a classical HSOS beyond day 21, or histologically proven HSOS; or based on hemodynamical or/and ultrasound evidence of HSOS plus any two of four features including bilirubin $\geq 2 \mathrm{mg} / \mathrm{dL}$, painful hepatomegaly, weight gain $>5 \%$, or ascites.

Since there are no international consensuses on the diagnosis of PA-HSOS, we adopted the recommendations from EBMT for HSCT-induced HSOS in a combination of the drug-induced liver injury 
(DILI) diagnosis with some minor modifications for all PA-HSOS ${ }^{4}$. Such modifications are supported by several recent peer-reviewed publications ${ }^{6,11}$, which included the confirmed history of PA intake and one of the following criteria: 1) ultrasound/enhanced computed tomography (CT)/magnetic resonance imaging (MRI) evidence of HSOS plus any two of four features including any levels of bilirubin elevation, painful hepatomegaly, weight gain $>5 \%$, or ascites; 2) histologically proven HSOS with features such as swelling, damage, and shedding of hepatic sinusoid endothelial cells in hepatic acinus zone III, or significant dilation and congestion of hepatic sinusoids ${ }^{12}$; 3) Roussel Uclaf Causality Assessment Method (RUCAM) score $\geq 6^{4}$. Our modifications were mainly the replacement of ultrasound with enhanced CT/MRI because these imaging studies had widely accepted diagnostic value 5, 13-15, and replacing the criteria of bilirubin level $\geq 2 \mathrm{mg} / \mathrm{dL}$ with any levels of elevation, because $40 \%$ to $50 \%$ of the patients with a confirmed diagnosis of PA-HSOS had an elevation of bilirubin levels but lower than 2 $\mathrm{mg} / \mathrm{dL}$ in recent studies ${ }^{6,11}$.

Study subjects were eligible for enrollment based on the following criteria: 1) age $\geq 18$ years old; 2) met the aforementioned diagnosis criteria for PA-HSOS. The major exclusion criteria were: 1) patient with coexisting other liver diseases including chronic viral hepatitis B or $\mathrm{C}$, alcoholic liver disease, autoimmune liver diseases, hepatocellular carcinoma, or Budd-Chiari syndrome; 2 ) a serious disease of heart, lung, or kidney; 3 ) patient with incomplete follow up data for the extraction of clinical variables.

\section{The standard care and data collection}

The standard care in the participating sites for PA-HSOS was mainly supportive treatment including nutrition supplements, albumin transfusion, and the use of adenosylmethionine or antibiotics if necessary. Treatment selections including anticoagulant, TIPSS, or liver transplantation were based on the discretion of the providers. Clinical data were collected at the three-time points including the first visit with the diagnosis of PA-HSOS as the baseline, at three months from the baseline visit as the primary outcome assessment point, and the 36 months from the baseline for the secondary outcome assessment. Data of patients' demographics and clinically relevant parameters were extracted from electronic and/or paper charts from the first visit related to the PA-HSOS until the last follow-up visit at the participating centers. We considered the following as the relevant clinical data: medical history, symptoms, signs, laboratory tests, liver pathology reports, CT/MRI results, treatment records, follow-up progress notes. In addition, survival data were collected by phone call communication with patients and their family members. The model of end-stage liver disease (MELD) scores, CTP-Turcotte-Pugh (CTP) scores, and sequential organ failure assessment (SOFA) were calculated by using the baseline data.

\section{Outcome assessments}

The primary outcome assessment was the survival in 3 months, defined by the percentage of patients who survived without liver transplantation at the end of 3 months follow up. The secondary assessments were the following: 1) predictors for the short-term survival by comparing the baseline factors and interventions between patients who survived and those who were transplanted or expired at the end of 3 
months; 2) The efficacy and safety of anticoagulation treatment by assessing the survival rates with versus without the intervention and the frequency of bleeding-related complications. Lastly, we attempt to assess two exploratory outcomes: 1 ) long-term survival rates by analyzing the percentage of patients who survived at 36 months without liver transplantation; 2) predictors for long-term survival by comparing the baseline factors and interventions between patients who survived and those who were transplanted or expired at the end of 36 months.

\section{Statistical analysis}

Clinical characteristics of subjects were compared between the survival and non-survival groups. Descriptive values were expressed as mean \pm standard deviation (SD) or medians and interquartile ranges (IQR), depending on the underlying distribution of the data. Student t-test or Mann-WhitneyWilcoxon test was used to assess continuous variables according to value distributions. Frequencies and percentages were used to summarize categorical variables and data were compared by using Pearson's Chi-square or Fisher's exact tests when appropriate. The $\chi^{2}$ test was also used if indicated. The Statistical Package for Social Science for Windows, version 19.0 (SPSS Inc., IBM, New York) was used for data analyses. All comparison tests between the two groups were 2-tailed with a $95 \%$ confidence interval, except the outcome comparison between the anticoagulation group and routine treatment group which used a one-tailed test. The Kaplan-Meier method was used to analyze the survival difference, followed by a log-rank test for comparing the Kaplan-Meier estimates. Multivariable Cox regression analyses were performed to assess the independent predictors for patient survival. The statistical significance was set at the $p$-value of $<0.05$.

\section{Results}

\section{Study patients}

During the enrollment period of 6 years, 63 patients were diagnosed with PA-HSOS and screened. Among them, 14 patients were excluded from the study because they were classified as co-existed with other liver diseases, heavy drinking, lack of data, or lost follow-up. The patient deposition including the reasons for enrollees who took the PA-related products is shown in Figure 1. Of 49 patients enrolled, all had a history of taking Tu-San-Qi orally before the onset of HSOS. Eighty-four percent of patients took PA product in herbal tea $(n=26 / 49)$ or decoction $(n=15 / 49)$, whereas fewer patients were on the formula of herbal liquor $(n=5)$ or tablets $(n=3)$. The median (IQR) time from initiating Tu-San-Qi to the onset of HSOS was 4 months (1-12months). When stratified with the duration of taking PA-related product for 1-2, 3-5, 6-11, and $\geq 12$ months, the percentage of patients who took the product with each time segment was $32.7 \%, 49.0 \%$, $65.3 \%$, and $85.7 \%$, respectively.

At the onset of PA-HSOS, patients' median age (IQR) was 60 (50-67) years and $49 \%$ of them were male. Their clinical features and laboratory variables are presented in Table 1. The top three clinical manifestations were ascites (98.0\%), abdominal distension (91.8\%), and hepatomegaly (51.0\%). When 
the severity of the disease was assessed, the median (IQR) scores for CTP, SOFA, and MELD were 9 (711), 3 (2-5), and 10 (5-18), respectively. Stratified by CTP scores for the severity of liver disease of the enrollees, the proportion of patients with CTP class A, B, and C were 8.2\% (4/49), 42.8\% (21/49), and $49.0 \%(24 / 49)$, respectively. Fourteen patients had available liver biopsy results during the recovery period. All presented features supported the diagnosis, which included concentric intimal fibrosis in central veins, congestion of hepatic sinusoids in hepatic acinus zone III around the central veins, or hepatic necrosis (Figure 2).

\section{Patient outcomes at three months}

None of our patients had the opportunity to receive liver transplantations or TIPSS in our cohort. The short-term survival rate at 3 months was $86 \%$ (42/49). Stratified by the baseline CTP scores, the survival rates at three months for patients with CTP class A, B, and C were $100.0 \%, 100.0 \%, 70.8 \%$, respectively. The leading causes of death were liver failure $(n=5)$, infection $(n=4)$, and multiple organ failure $(n=2)$. Although $37 / 49$ (76\%) of patients survived, $12 / 37$ patients developed liver-related complications including portal hypertension $(n=6)$, esophageal and/or gastric varices $(n=4)$, and ascites $(n=2)$. When the baseline variables of the survival group were compared with those of patients who expired (Table 1), patients who survived had significantly lower levels of AST, bilirubin, blood urea nitrogen (BUN), white blood cell (WBC) count, lower international normalized ratio (INR), and higher levels of fibrinogen or albumin. In addition, the survival group had significantly lower median CTP scores $(p=0.001)$ and SOFA scores $(p=0.004)$, although the MELD scores were similar between the two groups. During the 3 months follow-up, $14.3 \%$ and $61.9 \%$ of patients received anticoagulants in the non-survival and the survival groups, respectively $(p=0.03)$.

\section{Predictors for the short-term survival}

In a multivariate Cox model for delineating the independent factors for predicting the 3-month survival, we analyzed factors that significantly differed between the aforementioned two groups in the univariate analysis (Supplementary Table 1). The regression analyses indicated that the CTP class $C$ at the baseline and the use of anticoagulants during the first three months were the only negative $(H R=2.54)$ and positive ( $H R=0.11)$ independent predictors for the 3-month survival, respectively (Table 3$)$. When patients were classified as CTP class $\mathrm{C}$ at the onset of PA-HSOS, the risk of death increased by 1.25 folds when compared to that of patients who were classified as CTP class A or B.

\section{The effect and safety of anticoagulation}

Totally $27 / 49$ patients $(55.1 \%$ ) received anticoagulants in the first three months with $26 / 27$ and $1 / 27$ in the survival group and non-survival group, respectively. Their baseline features are showed in Supplementary Table 2. Among them, 21 were treated with low molecular weight heparin (LMWH), 2 with warfarin, and 4 with standard heparin. The overall survival rate at 3-months for patients treated with anticoagulants was $96.3 \%$. The survival rates in patients who received LMWH, warfarin, and heparin were $90.5 \%(19 / 21), 100 \%(2 / 2)$, and $75 \%(3 / 4)$ in 3 months, respectively $(p=0.20)$. Stratifying by CTP class, 
the 3-month survival rates were $100 \%(13 / 13)$ and $93 \%(13 / 14)$ in the CTP class A or B group and CTP class $C(p=0.52)$, respectively.

When compared to the anticoagulant treated-group, the survival rate for patients who received supportive therapy without anticoagulant $(n=22)$ was significantly lower $\left(96 \%[26 / 27]\right.$ vs. $59.1 \%[13 / 22] ; \chi^{2}=5.821$; $p=0.02$ ) in three months. Further subset analyses in the patients who did not receive anticoagulants indicated that those with CTP class $C$ had a significantly higher case-fatality rate when compared to that of patients with CTP class A or B $(60 \%$ vs. $0 \%, p<0.001)$. In contrast, anticoagulation therapy decreased the risk of death by $89 \%$ in the treatment method comparison. The risk reduction was even more profound in the subgroup patients with CTP class $\mathrm{C}$ (Table 2 ) because the 3-month survival rates decreased from $93 \%$ to $40 \%$ when patients were treated without anticoagulant $(p=0.009)$. The comparison of the longitudinal data in Figure 3 illustrated the survival rates at the time points of 1,3,6, and 36 months between the two groups. The Kaplan-Meier curves indicated a significantly better probability of survival in patients who were treated with any anticoagulant when compared with those who received supportive therapy without anticoagulant $(Z=6.46, p=0.01)$. In terms of safety data, none of the patients who received anticoagulant therapy had a complication of hemorrhage or other adverse events reported to be associated with the anticoagulant.

\section{Outcomes at 36 months and predictors}

At 36 months follow-up, the survival rate of the entire cohort was $75.5 \%$. Those who were expired included 2 and 3 patients in the anticoagulation therapy group and the non-anticoagulant treated group, respectively. All expired patients had baseline CTP class C except one with CTP class B in the nonanticoagulant treated group. When compared patients who survived at 36 months versus those who did not, the non-survival group were older, with significantly higher baseline levels of AST, bilirubin, prolonged $\mathrm{PT}$, creatinine, BUN, increase in the INR or WBC, or lower levels of fibrinogen or albumin. In addition, these patients had significantly higher median CTP scores $(p<0.001)$ and SOFA scores $(p=0.001)$. However, the MELD scores at the baseline did not differ between the two groups (Supplementary Table 3).

Among those who received anticoagulation treatment, the survival rates were $100 \%$ and $79 \%$ in patients with CTP class A or B and CTP class $C$, respectively $(p=0.12)$. In contrast, in patients who did not receive anticoagulants, the survival rates were significantly lower in patients with CTP class $C$ versus those with CTP class A or B $(20 \%$ [2/10] vs $91.7 \%$ [11/12]; $p<0.001)$. Patients with a baseline CTP class $C$ had the risk of death increase by 1.29 folds when compared to that of those with CTP class A or B (Supplementary Table 4). Anticoagulation treatment decreased the risk of death by $84.3 \%$ at 36 months. The survival rate of patients with CTP class $C$ was significantly higher in the anticoagulation group than that of patients who did not receive the treatment $(p=0.007)$. However, the survival rates were similar between the two groups ( $p=0.48)$, when patients were classified as CTP class A or B.

The baseline variables associated with survival of 36 months in univariate analyses were presented in Supplementary Table 1. In a multivariate Cox model (Supplementary Table 5), CTP class C $(H R=2.29)$ and 
anticoagulant therapy $(\mathrm{HR}=0.16)$ were the only independent negative and positive factors for predicting 36-month survival, respectively. These independent prognostic baseline factors were identical to those found in the analyses of 3-month survival.

\section{Discussion}

PA-HSOS is a rare form of DILI in Asia, although in China herbal and dietary supplementary is a leading cause of DILI $\left(26.8 \%\right.$ of DILI) ${ }^{16}$. The case-fatality rate was estimated to be $>80 \%$ in patients with severe $\mathrm{HSOS}^{7}$. Although Zhu et al. recently summarized the clinical presentations of 124 individual patients with PA-HSOS from 91 case reports in different studies, cohort data are lacking on the prognostic model and risk factor analyses to help clinicians with identifying those with poor outcomes for early interventions, such as anticoagulation therapy, TIPSS, or transferring patients to liver transplant centers. We retrospectively enrolled 49 patients with PA-HSOS from 5 centers in different regions. We observed a 3month survival rate of $86 \%$, which was decreased to $75 \%$ at the 36 -month follow-up. The CTP class $C$ at baseline or lack of anticoagulation therapy during the first three months were the independent negative predictors for both 3- and 36-month survival. To our knowledge, the current study is the first one by far to address the risk factors on survival in this unique disease. Our study filled in the aforementioned data gap.

The diagnosis of PA-HSOS remains a challenge that requires a detailed history and clinical investigation ${ }^{17}$. Several studies have reported the features of PA-HSOS ${ }^{5,13,17}$. Patients often presented with profound fatigue, jaundice, abdominal pain or distention, hepatomegaly, ascites, and edema. Laboratory findings frequently included elevations in bilirubin, ALT, AST, ALP, bilirubin, and GGT levels ${ }^{5,17}$. Typical imaging examinations showed ascites, hepatomegaly, and splenomegaly ${ }^{13}$. Our study concurred with their findings. Our patients showed the profile of ascites (98.0\%), abdominal distension (91.8\%), and hepatomegaly $(51.0 \%)$, bilirubin levels $>34.2 \mu \mathrm{mol} / \mathrm{L}(41 \%)$. However, the aforementioned features were similar to those of patients with decompensated liver diseases from other etiologies ${ }^{17}$, which posed challenges in the clinical setting and required liver biopsy to confirm the diagnosis of HSOS in some cases. Lastly, performing serum PA level has a great value of confirming the cause of DILI. However, none of our patients had the access to the test because it was not available in any of the study sites.

Additionally, liver transplants or TIPSS were not performed in our cohort.

Fewer studies had explored the impact of anticoagulation therapy in these patients, which had great discrepancies with the short-term survival rates ranging from $60 \%$ to $87 \% 6,8$. However, these studies failed to demonstrate a significant benefit of anticoagulant for patients with severe PA-HSOS for the following reasons: 1) patients were not stratified by the severity of PA-HSOS for comparison in the study by Wang et al $^{7}$. 2) In Zhuge's study ${ }^{9}$, patients in the supportive group had significantly severe PA-HSOS at baseline than that in the anticoagulant group, which also mixed with patients who received both TIPSS and anticoagulant treatment. Stratifying by CTP class, our data suggested that anticoagulant therapy not only benefits these patients but also significantly reduced the case-fatality rates in patients with CTP 
class $\mathrm{C}$ at baseline. There was a marked increase in survival with the therapy when compared to the supportive treatment ( $89 \%$ vs. $59.1 \%, p=0.02)$, particularly among patients with CTP class $C(H R=2.54)$. Our safety data on anticoagulants were consistent with those in published studies.

Several limitations are noted in our study, which included the retrospective design with limited capacity to identifying confounding factors affecting survival. In addition, it is possible to miss other independent prognostic factors because of the feasibility in real-world practices, such as measuring PA serum concentration. Further prospective studies are needed to verify our findings. Furthermore, patients were all Chinese and received standard care in China, our findings require validation before generalizing to other populations with different settings. Despite the limitations, the current study advances our knowledge on how to select patients for early intervention and provides preliminary results to support anticoagulant therapy for this special population.

In conclusion, our multicenter study observed that the 3-month and 36-month survival rates of patients with PA-HSOS were $86 \%$ and $75 \%$, respectively. Compared to the CTP class A or B, class C at baseline independently predicted lower survival rates at both 3 and 36 months without TIPSS and/or liver transplantation. Thus, patients with CTP class $\mathrm{C}$ should receive further interventions on top of supportive therapy, including anticoagulants or referral to the transplant center. Anticoagulation therapy may offer a benefit for all patients without a major safety concern at present, particularly among those with the CTP class $\mathrm{C}$.

\section{Abbreviations}

ALP alkaline phosphatase

ALT alanine aminotransferase

AST aspartate aminotransferase

BUN blood urea nitrogen

$\mathrm{Cl} \quad$ confidence interval

DBIL direct bilirubin

GGT gamma-glutamyl transpeptidase

INR international normalized ratio

LMWH low molecular weight heparin

PA-HSOS Pyrrolizidine alkaloids induced hepatic sinusoidal obstruction syndrome PT prothrombin time 
RBC red blood cell

TIPSS transjugular intrahepatic portosystemic shunt

TT thrombin time

WBC white blood cell

\section{Declarations}

\section{ACKNOWLEDGMENTS}

We thank Ms. Celeste Zhang for her editorial assistance in proofreading the manuscript.

\section{Funding}

The study was conducted by authors without funding support.

\section{Conflicts of interest/Competing interests (include appropriate disclosures)}

Xiaofei Du, Zhenli Liu, Haibin Yu, Yu Wang, Zhengsheng Zou, Hongshan Wei, Jing Liang, Daokun Yang, Yali Liu, Jing Zhang, and Calvin Pan have no competing interest to disclose.

\section{Ethics approval (include appropriate approvals or waivers)}

The study protocol was approved by the individual Ethics Review Committee in all participated research sites including the leading institution, Beijing Youan Hospital (IRB approval number [2020]-233). Patients' informed consent was exempted by IRB.

\section{Consent to participate (include appropriate statements)}

Patients' data were retrospectively collected and de-identified before performing data analyses. Patients' informed consent was exempted by IRB.

\section{Consent for publication (include appropriate statements)}

Patients' informed consent was exempted by IRB because the reports are the summary of the deidentification data.

\section{Availability of data and material (data transparency)}

The data that support the findings of this study are available from authors on reasonable request. Participant data without names and identifiers may be shared with other researchers after approval from the hospital authority and ethic committee. 
Not applicable

\section{Authors' contributions (optional: please review the submission guidelines from the journal whether statements are mandatory)}

Guarantor of the article: Jing Zhang

The study was designed by Dr. Jing Zhang with inputs from Dr. Calvin Q. Pan; Dr. Zhang also managed the study with assistance from Dr. Yali Liu; Drs. Yu Wang, Zhengsheng Zou, Hongshan Wei, Jing Liang, and Daokun Yang collected the data; Drs. Zhenli Liu and Xiaofei Du analyzed the data. The manuscript was written by Calvin Q. Pan, Xiaofei Du, Zhenli Liu, and Haibin Yu. Drs Calvin Q.Pan and Jing Zhang performed the critical revisions including interpretation of data and address the editorial comments. All the authors vouch for the veracity and completeness of the data and analyses presented. The final version of the manuscript has been reviewed and approved by the authors.

\section{References}

1. European Association for the Study of the Liver. Electronic address. EASL Clinical Practice Guidelines: Vascular diseases of the liver. J Hepatol 2016;64:179-202.

2. Mohty M, Malard F, Abecassis M, et al. Sinusoidal obstruction syndrome/veno-occlusive disease: current situation and perspectives-a position statement from the European Society for Blood and Marrow Transplantation (EBMT). Bone Marrow Transplant 2015;50:781-9.

3. Rubbia-Brandt L. Sinusoidal obstruction syndrome. Clin Liver Dis 2010;14:651-68.

4. European Association for the Study of the Liver. Electronic address, Clinical Practice Guideline Panel C, Panel $m$, et al. EASL Clinical Practice Guidelines: Drug-induced liver injury. J Hepatol 2019;70:1222-1261.

5. Yang XQ, Ye J, Li X, et al. Pyrrolizidine alkaloids-induced hepatic sinusoidal obstruction syndrome: Pathogenesis, clinical manifestations, diagnosis, treatment, and outcomes. World J Gastroenterol 2019;25:3753-3763.

6. Zhuge Y, Liu Y, Xie W, et al. Expert consensus on the clinical management of pyrrolizidine alkaloidinduced hepatic sinusoidal obstruction syndrome. J Gastroenterol Hepatol 2019;34:634-642.

7. Wang Y, Qiao D, Li Y, et al. Risk factors for hepatic veno-occlusive disease caused by Gynura segetum: a retrospective study. BMC Gastroenterol 2018;18:156.

8. Peng $C$, Zhang $X$, Zhang F, et al. Clinical efficacy and safety of anticoagulation therapy for Pyrrolizidine alkaloids-induced hepatic sinusoidal obstruction syndrome: a retrospective multicenter cohort study. Eur J Gastroenterol Hepatol 2020;32:1168-1178.

9. Zhuge YZ, Wang Y, Zhang F, et al. Clinical characteristics and treatment of pyrrolizidine alkaloidrelated hepatic vein occlusive disease. Liver Int 2018;38:1867-1874.

10. Mohty M, Malard F, Abecassis M, et al. Revised diagnosis and severity criteria for sinusoidal obstruction syndrome/veno-occlusive disease in adult patients: a new classification from the 
European Society for Blood and Marrow Transplantation. Bone Marrow Transplant 2016;51:906-12.

11. Roy Moulik N, Johnson I, Van Bruggen L, et al. Defibrotide treatment but not prophylaxis is useful in hepatic sinusoidal obstruction syndrome in children undergoing autologous stem cell transplant following high-dose chemotherapy: A single-center experience from the Royal Marsden Hospital, UK. Pediatr Blood Cancer 2020;67:e28677.

12. Bearman $\mathrm{SI}$, Anderson GL, Mori M, et al. Venoocclusive disease of the liver: development of a model for predicting fatal outcome after marrow transplantation. J Clin Oncol 1993;11:1729-36.

13. Zhou H, Wang YX, Lou HY, et al. Hepatic sinusoidal obstruction syndrome caused by herbal medicine: CT and MRI features. Korean J Radiol 2014;15:218-25.

14. Kan X, Ye J, Rong X, et al. Diagnostic performance of Contrast-enhanced CT in Pyrrolizidine Alkaloids-induced Hepatic Sinusoidal Obstructive Syndrome. Sci Rep 2016;6:37998.

15. Wang C, Wu X, Xie W, et al. Quantitative Analysis of CT Images in Patients with Pyrrolizidine AlkaloidInduced Sinusoidal Obstruction Syndrome. Sci Rep 2019;9:2179.

16. Shen T, Liu Y, Shang J, et al. Incidence and Etiology of Drug-Induced Liver Injury in Mainland China. Gastroenterology 2019;156:2230-2241 e11.

17. Zhu L, Zhang CY, Li DP, et al. Tu-San-Qi (Gynura japonica): the culprit behind pyrrolizidine alkaloidinduced liver injury in China. Acta Pharmacol Sin 2020.

\section{Tables}

Table 1 Baseline characteristics of PA-HSOS patients with following-up for 3 months 


\begin{tabular}{|c|c|c|c|c|c|}
\hline Characteristic & Total & $\begin{array}{c}\text { Survival group } \\
(\mathrm{n}=42)\end{array}$ & $\begin{array}{l}\text { Non-survival } \\
\text { group }(\mathrm{n}=7)\end{array}$ & $\begin{array}{c}\text { Statistic } \\
\text { value }\end{array}$ & $\mathrm{P}$ \\
\hline Age (years) & $\begin{array}{l}\text { 60.00(50.00- } \\
67.00)\end{array}$ & $\begin{array}{l}59.50(49.00- \\
67.00)\end{array}$ & $\begin{array}{l}65.00(60.00- \\
67.00)\end{array}$ & $Z=-1.07$ & 0.28 \\
\hline Male, n (\%) & $24(48.98)$ & $19(45.24)$ & $5(71.43)$ & $c^{2}=1.65$ & 0.19 \\
\hline $\begin{array}{l}\text { Time of PAs taken } \\
\text { (months) }\end{array}$ & $\begin{array}{l}4.00(1.00- \\
12.00)\end{array}$ & $\begin{array}{l}3.50(1.00- \\
12.00)\end{array}$ & $6.00(1.00-14.00)$ & $Z=-0.68$ & 0.53 \\
\hline Ascites, n (\%) & $48(98.00)$ & $41(97.62)$ & $7(100.00)$ & $c^{2}=0.17$ & 0.86 \\
\hline $\begin{array}{l}\text { Abdominal distension, } \mathrm{n} \\
\text { (\%) }\end{array}$ & $45(91.84)$ & $38(90.48)$ & $7(100.00)$ & $c^{2}=0.73$ & 0.53 \\
\hline Hepatomegaly, n (\%) & $25(51.02)$ & $21(50.00)$ & $4(57.14)$ & $c^{2}=0.12$ & 0.52 \\
\hline $\begin{array}{l}\text { Lower limbs edema, } \mathrm{n} \\
(\%)\end{array}$ & $24(48.98)$ & $21(50.00)$ & $3(42.86)$ & $c^{2}=0.12$ & 0.52 \\
\hline Splenomegaly, n (\%) & $21(42.86)$ & $18(42.86)$ & $3(42.86)$ & $c^{2}=0.00$ & 0.65 \\
\hline $\begin{array}{l}\text { Right upper quadrant } \\
\text { pain, } \mathrm{n}(\%)\end{array}$ & $11(22.45)$ & $8(19.05)$ & $3(42.86)$ & $c^{2}=0.83$ & 0.18 \\
\hline $\begin{array}{l}\text { Esophagogastric } \\
\text { varices, } \mathrm{n}(\%)\end{array}$ & $21(42.86)$ & $19(45.24)$ & $2(28.57)$ & ${ }^{2}=0.17$ & 0.35 \\
\hline $\begin{array}{l}\text { Abdominal infection, } \mathrm{n} \\
(\%)\end{array}$ & $8(16.33)$ & $4(9.52)$ & $4(57.14)$ & $c^{2}=6.78$ & 0.009 \\
\hline $\begin{array}{l}\text { Hepatic } \\
\text { encephalopathy, } \\
\text { n (\%) }\end{array}$ & $5(10.20)$ & $2(4.76)$ & $3(42.86)$ & ${ }^{2}=5.80$ & 0.02 \\
\hline WBC $\left(10^{9} / \mathrm{L}\right)$ & $\begin{array}{l}6.40(3.85- \\
8.80)\end{array}$ & $6.05(3.65-7.95)$ & $8.50(6.90-12.00)$ & $Z=-2.20$ & $0.03^{*}$ \\
\hline $\operatorname{RBC}\left(10^{12} / \mathrm{L}\right)$ & $\begin{array}{l}4.76(3.99- \\
5.28)\end{array}$ & $4.73(4.03-5.24)$ & $4.82(3.08-5.52)$ & $Z=-0.10$ & 0.92 \\
\hline Platelet $\left(10^{9} / \mathrm{L}\right)$ & $\begin{array}{l}97.00(71.00- \\
143.50)\end{array}$ & $\begin{array}{l}106.50(71.50- \\
161.75)\end{array}$ & $\begin{array}{l}84.00(60.00- \\
101.00)\end{array}$ & $Z=-1.46$ & 0.15 \\
\hline ALT (U/L) & $\begin{array}{l}28.00(15.05- \\
52.80)\end{array}$ & $\begin{array}{l}28.40(15.75- \\
52.70)\end{array}$ & $\begin{array}{l}28.00(15.00- \\
53.30)\end{array}$ & $Z=-0.16$ & 0.88 \\
\hline AST (U/L) & $\begin{array}{l}51.00(36.00- \\
76.90)\end{array}$ & $\begin{array}{l}46.45(35.50- \\
71.90)\end{array}$ & $\begin{array}{l}79.00(59.70- \\
162.40)\end{array}$ & $Z=-2.04$ & $0.04^{*}$ \\
\hline $\mathrm{ALP}(\mathrm{U} / \mathrm{L})$ & $\begin{array}{l}119.8(92.25- \\
162.50)\end{array}$ & $\begin{array}{l}119.40(92.88- \\
145.05)\end{array}$ & $\begin{array}{l}170.90(80.80- \\
207.00)\end{array}$ & $Z=-0.63$ & 0.53 \\
\hline GGT (U/L) & $\begin{array}{l}92.90(62.50- \\
133.95)\end{array}$ & $\begin{array}{l}91.25(62.75- \\
127.98)\end{array}$ & $\begin{array}{l}109.00(41.90- \\
272.00)\end{array}$ & $Z=-0.86$ & 0.39 \\
\hline Bilirubin $(\mu \mathrm{mol} / \mathrm{L})$ & $\begin{array}{l}41.20(22.07- \\
83.85)\end{array}$ & $\begin{array}{l}35.10(20.60- \\
64.28)\end{array}$ & $\begin{array}{l}101.80(76.80- \\
214.00)\end{array}$ & $Z=-3.06$ & $0.002^{*}$ \\
\hline DBIL $(\mu \mathrm{mol} / \mathrm{L})$ & $\begin{array}{l}21.00(12.00- \\
52.35)\end{array}$ & $\begin{array}{l}15.10(11.75- \\
33.17)\end{array}$ & $\begin{array}{l}84.90(47.90- \\
171.30)\end{array}$ & $Z=-3.29$ & $0.001^{*}$ \\
\hline Albumin $(\mathrm{g} / \mathrm{L})$ & $\begin{array}{l}33.00(27.50- \\
36.30)\end{array}$ & $\begin{array}{l}34.50(29.68- \\
37.73)\end{array}$ & $\begin{array}{l}25.00(22.80- \\
28.00)\end{array}$ & $Z=-3.45$ & $0.001^{*}$ \\
\hline BUN (mmol/L) & $\begin{array}{l}5.70(4.00- \\
9.30)\end{array}$ & $5.25(4.00-7.20)$ & $11.30(6.70-14.30)$ & $Z=-2.36$ & 0.02 \\
\hline Creatinine $(\mu \mathrm{mol} / \mathrm{L})$ & $\begin{array}{l}\text { 69.20(52.65- } \\
92.20)\end{array}$ & $\begin{array}{l}\text { 68.85(51.75- } \\
86.35)\end{array}$ & $\begin{array}{l}90.00(66.00- \\
130.00)\end{array}$ & $Z=-1.74$ & 0.08 \\
\hline PT (S) & $\begin{array}{l}14.40(12.60- \\
19.20)\end{array}$ & $\begin{array}{l}14.10(12.47- \\
18.70)\end{array}$ & $\begin{array}{l}17.50(14.20- \\
27.60)\end{array}$ & $Z=-1.89$ & 0.06 \\
\hline INR & $\begin{array}{l}1.24(1.12- \\
1.64)\end{array}$ & $1.18(1.10-1.57)$ & $1.56(1.27-2.46)$ & $Z=-2.10$ & 0.03 \\
\hline Fibrinogen $(\mathrm{g} / \mathrm{L})$ & $\begin{array}{l}2.54(1.88- \\
3.03)\end{array}$ & $2.60(2.14-3.10)$ & $1.72(1.16-2.47)$ & $Z=-2.37$ & 0.02 \\
\hline
\end{tabular}




\begin{tabular}{llllll} 
TT $(\mathrm{S})$ & $16.60(14.65-$ & $15.95(14.60-$ & $19.50(16.20-$ & $\mathrm{Z}=-1.77$ & 0.08 \\
CTP score & $19.60)$ & $18.85)$ & $24.20)$ & & \\
SOFA score & $9(7-11)$ & $9(7-10)$ & $11(11-12)$ & $\mathrm{Z}=-3.30$ & $0.001^{*}$ \\
MELD score & $3.00(2.00-$ & $3.00(2.00-4.00)$ & $5.00(4.00-6.00)$ & $\mathrm{Z}=-2.87$ & $0.004^{*}$ \\
Treatment & $\begin{array}{l}4.50) \\
9.75(5.09-\end{array}$ & $10.01(4.32-$ & $9.05(7.74-11.85)$ & $\mathrm{Z}=-0.43$ & 0.67 \\
Routine treatment & $17.59)$ & $17.90)$ & & $\mathrm{C}^{2}=5.50$ & 0.03 \\
Anticoagulation & & & & \\
treatment & $22(44.90)$ & $16(38.10)$ & $6(85.71)$ & & \\
\hline
\end{tabular}

nine aminotransferase; AST, aspartate aminotransferase; ALP, alkaline phosphatase; BUN, blood urea 1; CTP, Child-Turcotte-Pugh; DBIL, direct bilirubin; GGT, gamma-glutamyl transpeptidase; HSOS hepatic al obstruction syndrome; INR, international normalized ratio; MELD, the model of end-stage liver disease; 'olizidine alkaloids; PT, thrombin time; RBC, red blood cell; SOFA, sequential organ failure assessment; TT, mbin time; WBC, white blood cell. Routine treatment means symptomatic and supportive treatment $\mathrm{g}$ nutrition supplements, albumin transfusion, and the use of adenosylmethionine or antibiotics if ry. Anticoagulation treatment means one anticoagulant, such as heparin, low molecular weight heparin, or 1 was used.

Table 2 Comparison of 3-month survival rates between patients treated with and without anticoagulation treatment

\begin{tabular}{|c|c|c|c|c|c|c|c|}
\hline & \multicolumn{3}{|c|}{ CTP class A or B } & \multicolumn{3}{|c|}{ CTP class C } & P1 \\
\hline & $\mathrm{n}$ & $\begin{array}{c}\text { Non-survival } \\
(\%)\end{array}$ & $\begin{array}{c}\text { Survival } \\
(\%)\end{array}$ & & $\begin{array}{c}\text { Non-survival } \\
(\%)\end{array}$ & $\begin{array}{c}\text { Survival } \mathrm{n} \\
(\%)\end{array}$ & \\
\hline $\begin{array}{l}\text { Anti-coagulation } \\
\text { treatment }\end{array}$ & 13 & $0(0.00)$ & $13(100.00)$ & 14 & $1(7.10)$ & $13(92.90)$ & 0.52 \\
\hline $\begin{array}{l}\text { Supportive } \\
\text { treatment }\end{array}$ & 12 & $0(0.00)$ & $12(100.00)$ & 10 & $6(60.00)$ & $4(40.00)$ & 0.003 \\
\hline Total & 25 & $0(0.00)$ & $25(100.00)$ & 24 & $7(29.20)$ & 17(70.80) & \\
\hline P2 & & - & & & & 0.009 & \\
\hline
\end{tabular}

CTP, Child-Turcotte-Pugh; P1, Survival rates comparison of CTP class (A or B) vs. CTP class C; P2, Survival rates comparison of the anticoagulation group vs. the supportive therapy group. Routine treatment means symptomatic and supportive treatment including nutrition supplements, albumin transfusion, and the use of adenosylmethionine or antibiotics if necessary. Anticoagulation treatment means one anticoagulant, such as heparin, low molecular weight heparin, or warfarin was used.

Table 3 Multivariate Cox regression analysis of risk factors for 3-month survival 


\begin{tabular}{llll}
\hline Factors & $\mathrm{P}$ & $\mathrm{HR}$ & $95 \%$ CI \\
\hline CTP class & 0.008 & 2.54 & $1.28-5.04$ \\
Anticoagulation treatment & 0.04 & 0.11 & $0.01-0.92$ \\
\hline
\end{tabular}

CTP, Child-Turcotte-Pugh; HR, hazard ratio; CI, confidence interval

\section{Figures}

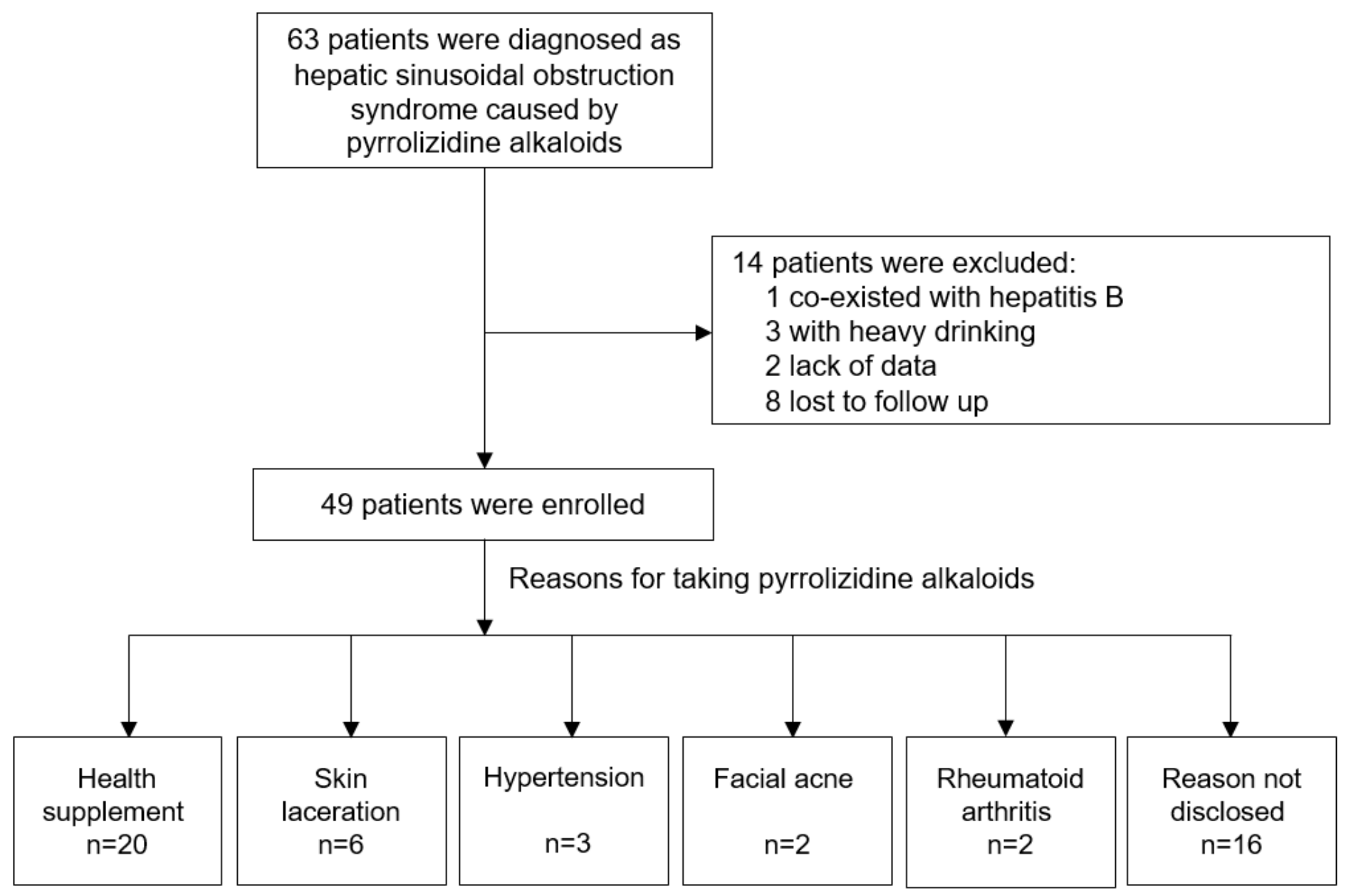

\section{Figure 1}

Flow chart of patient deposition 

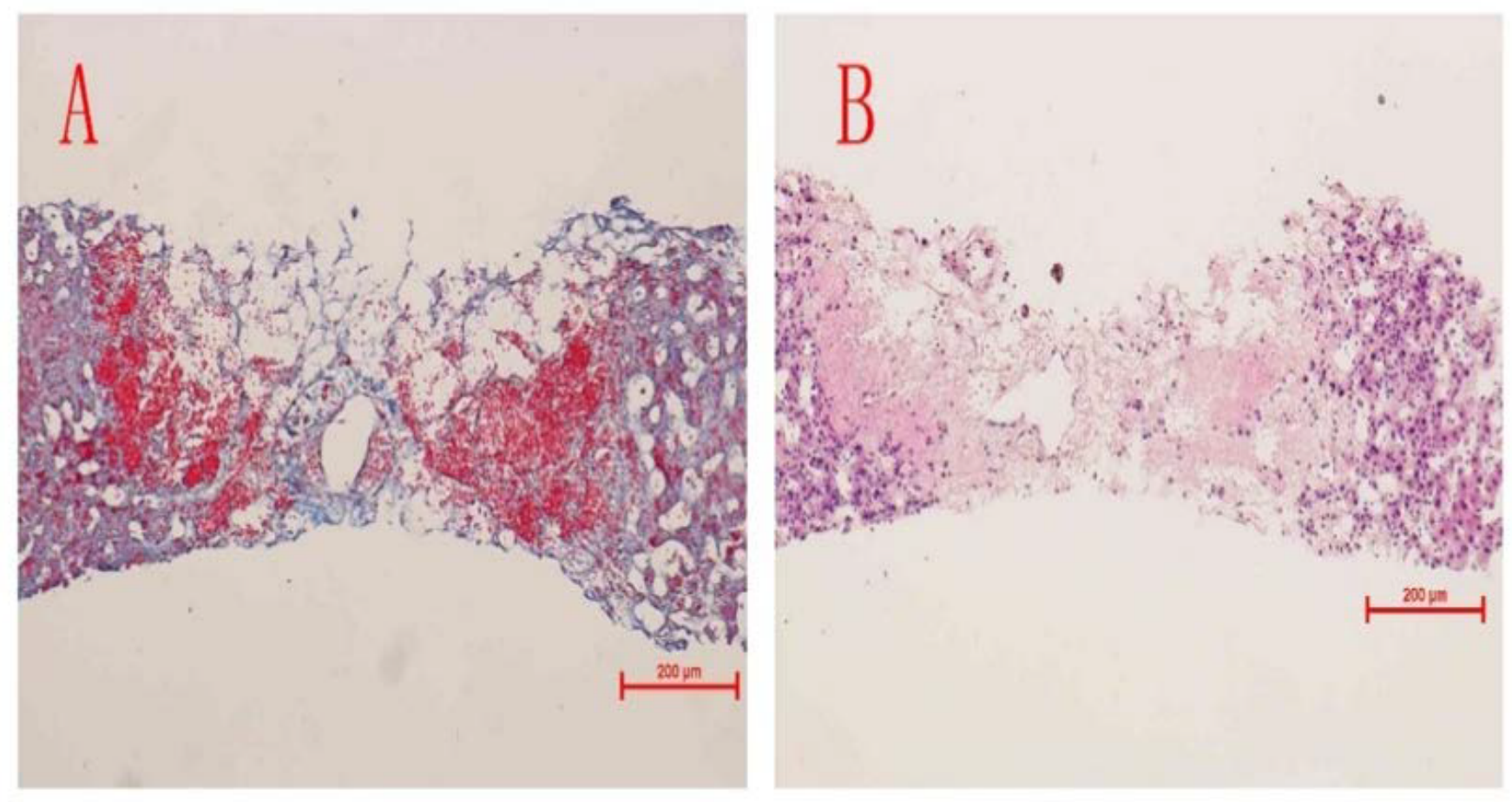

\section{Figure 2}

Pathological characteristics of sinusoidal obstruction syndrome induced by pyrrolizidine alkaloids A: Concentric intimal fibrosis in central veins (Masson's trichrome staining, original magnification of 100xpower) B: Congestion of hepatic sinusoids in hepatic acinus zone III around the central veins, hepatic damage, reticular fibrous network, and hepatic necrosis (HE staining, original magnification of 100xpower) 


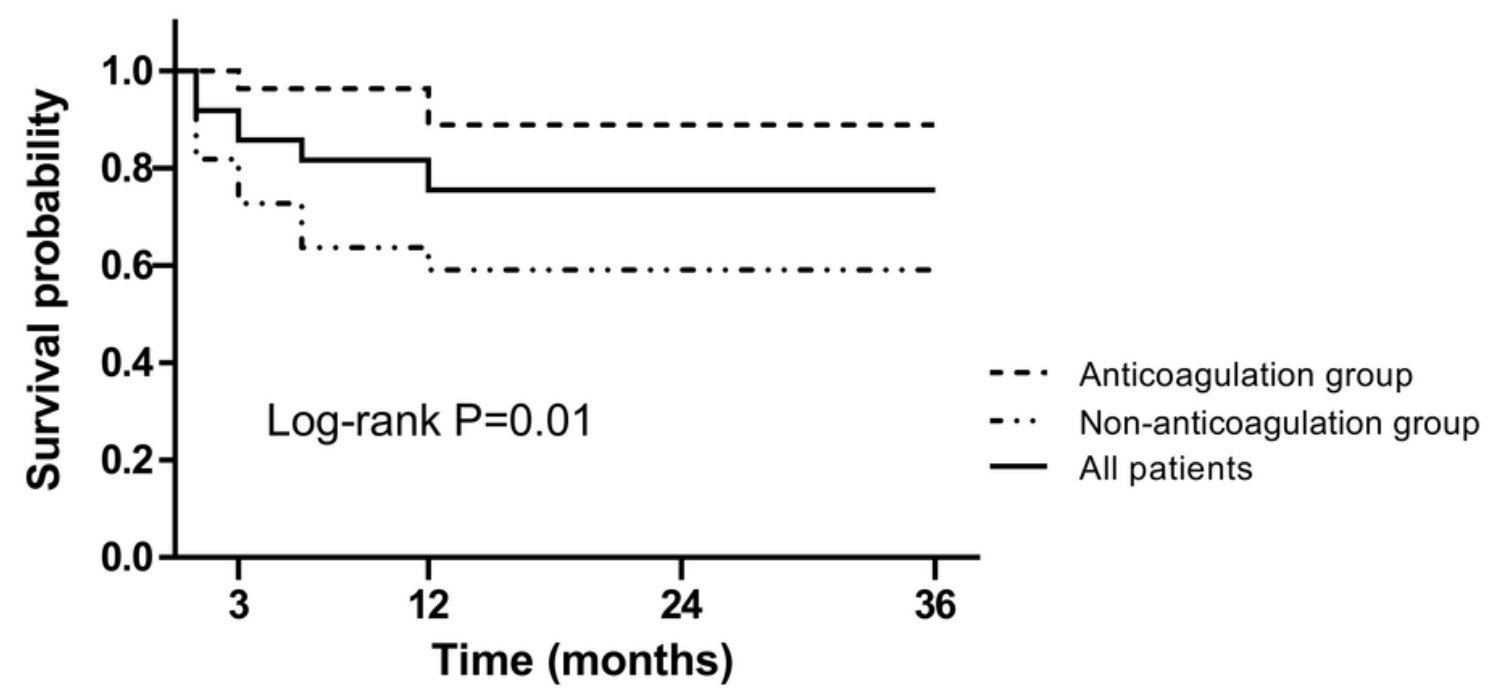

Number at risk by each time point

$\begin{array}{ccccc}\text { All patients } & 42 / 49 & 37 / 49 & 37 / 49 & 37 / 49 \\ \text { Anticoagulation } & 26 / 27 & 24 / 27 & 24 / 27 & 24 / 27 \\ & & & 13 / 22 & 13 / 22\end{array}$

Figure 3

Kaplan-Meier survival curves of study patients in 36 months The HR of anticoagulant therapy were 0.11 and 0.16 for 3 - and 36 -month survival, respectively

\section{Supplementary Files}

This is a list of supplementary files associated with this preprint. Click to download.

- SupplementaryTableS15.docx 